\title{
Analytical Wear Model of a Gerotor Pump without Hydrodynamic Effect*
}

\author{
Soon-Man KWON**, Mu Seong KIM*** and Joong-Ho SHIN** \\ ${ }^{* *}$ Dept. of mechanical design \& manufacturing engineering, Changwon National University \\ 9 Sarim-dong, Changwon, Kyongnam 641-773, Republic of Korea \\ E-mail:smkwon@changwon.ac.kr \\ *** Graduate School, Dept. of mechanical design \& mfg engineering, Changwon National University \\ 9 Sarim-dong, Changwon, Kyongnam 641-773, Republic of Korea
}

\begin{abstract}
The contact stress in a gerotor pump is addressed in this paper because the gerotor pump cannot be adjusted for wear. The first part of this paper presents a simple and exact rotor profile equations of the gerotor pump. In the second part of this paper, an explicit formula to avoid undercut in the inner-rotor of the gerotor pump is proposed by examining the minimum radius of curvature of the inner-rotor tooth profile on the convex section. It is found that undercut does not occur so long as the minimum value of the radius of curvature on the convex section is not less than zero. Next, the contact stresses without hydrodynamic effect between the inner and outer-rotors are evaluated through the calculation of the Hertzian contact stress in the rotor teeth. Based on the above results, we finally present the wear characteristics of $p_{H} V_{s}$ factor, which is proportional to the wear rate, between the rotors of the gerotor pump under quasi-static and dry contact conditions.
\end{abstract}

Key words: Gerotor Pump, Inner-Rotor, Outer-Rotor, Tooth Size Ratio, Radius of Curvature, Hertz Contact Stress, Wear Rate Proportional Factor

\section{Introduction}

Numerous applications in hydraulic and lubrication systems just require the circulation of the fluid. In such cases low noise emissions and little pressure ripples are more important than highly efficient transmission of energy.

The gerotor pump is ideal principle for such applications. Compared to conventional external gear pumps, the suction and pressure connection of the gerotor pump is axial to driving shaft. This also supports the compact construction. Due to the solid tooth shape, the gerotor pump is resistant to hydraulic and mechanical impact loads. The long durability of the gerotor pump is based on the relatively low speed between the internal and the external rotor. Furthermore, this pump is characterized by an extremely good smoothness and a low noise level.

Nevertheless, a disadvantage in the design of gerotor pumps is a lack of parts that can be adjusted to compensate for wear in the rotor set, and as a consequence, it causes sharp reduction of efficiency. Therefore, when the rotors become worn they must simply be replaced ${ }^{(1)}$. The main difficulty in calculating the contact stress is to determine the force that is transmitted through each contact point. Since there are many contact points, at any instant, the problem is statically indeterminate, and it is necessary to consider the tooth deformation at the contact points.

This paper presents geometric and kinematic aspects that constitute a premise to the modeling and simulation of gerotor pumps. Gearing of a gerotor pump's rotor set with

*Received 5 Oct., 2007 (No. 07-0614) [DOI: 10.1299/jamdsm.2.230] 
outer-rotor having one gear tooth more than inner-rotor is analyzed by the principle of the instantaneous center ${ }^{(2)}$.

Based on developed analytical expressions, we finally present the wear characteristics of $p_{H} V_{s}$ factor, which is proportional to the wear rate, between the rotors of the gerotor pump under quasi-static and dry contact conditions.

\section{Rotor profile equations}

The number of teeth of the inner-rotor is always one less than the outer-rotor. The outer and inner-rotors have respectively $N$ and $(N-1)$ teeth. We can choose any shape for the outer-rotor teeth, and the inner-rotor is then generated conjugate to the outer-rotor. We describe here only the outer-rotor having $N$ arcs of circle in the placement of $R$ from its center with the radius of $R_{r}$. The following is a brief summary of the rotor geometry, which is described in more detail in Refs. (2) and (3).

Before deriving the profile equations of the inner and the outer-rotors, four coordinate systems corresponding to the rotor set should be defined as shown in Fig. 1: two stationary reference systems $S_{2 f}\left(x_{2 f}, y_{2 f}\right)$ and $S_{3 f}\left(x_{3 f}, y_{3 f}\right)$, and two mobile reference systems $S_{2}\left(x_{2}, y_{2}\right)$ and $S_{3}\left(x_{3}, y_{3}\right)$.

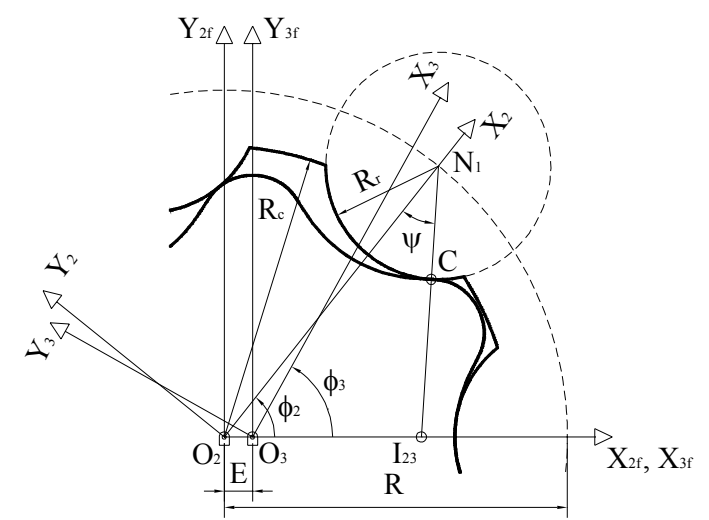

Fig.1 Four reference systems for the profile derivation of inner-rotor

The outer-rotor turns about $\mathrm{O}_{2}$, and the inner turns about its center $\mathrm{O}_{3}$, the angular velocity ratio being $(N-1): N$. If $E$ is the distance between $\mathrm{O}_{2}$ and $\mathrm{O}_{3}$, then pitch point $I_{23}$ lies on $\mathrm{O}_{2} \mathrm{O}_{3}$ a distance $r_{2}=E N$ from $\mathrm{O}_{2}$ and $r_{3}=E(N-1)$ from $\mathrm{O}_{3}$. If $\phi$ is the counterclockwise rotation of the outer-rotor relative to the inner-rotor, then the counterclockwise rotations $\phi_{2}$ and $\phi_{3}$ of the outer and inner-rotors are given by

$$
\phi_{2}=-(N-1) \phi, \phi_{3}=-N \phi
$$

For the homogeneous coordinate transformation from the contact point of ${ }^{2} C$ in $S_{2}$-reference system to that of ${ }^{3 f} C$ in $S_{3 f}$-reference system, the following matrix equation is defined:

$$
{ }^{3 f} C={ }^{3 f} M_{3}{ }^{3} M_{2 f}{ }^{2 f} M_{2}{ }^{2} C
$$

where the matrix ${ }^{i} M_{j}$ describes transformation from $S_{j}$-system to $S_{i}$-system, and 


$$
{ }^{2} C=\left[\begin{array}{llll}
R-R_{r} \cos \psi & -R_{r} \sin \psi & 0 & 1
\end{array}\right]^{T}
$$

Here the superscript $T$ in Eq. (3) means the transpose of the matrix.

The resulting expression of Eq. (2) is

$$
\begin{aligned}
& { }^{3 f} C_{x}=R \cos \left(\phi+\theta_{3}\right)-\left(R_{r}+\delta_{t}\right) \cos \left(\phi+\psi+\theta_{3}\right)-E \cos \left(N \phi+\theta_{3}\right) \\
& { }^{3 f} C_{y}=R \sin \left(\phi+\theta_{3}\right)-\left(R_{r}+\delta_{t}\right) \sin \left(\phi+\psi+\theta_{3}\right)-E \sin \left(N \phi+\theta_{3}\right)
\end{aligned}
$$

where

$$
\psi=-\arctan \left[\frac{\sin (N-1) \phi}{\mu-\cos (N-1) \phi}\right]
$$

and $\theta_{3}$ is the rotation angle of the inner-rotor, also the parameter of $\mu=R / r_{2}$ is referred to as the trochoid ratio. It should be noted that most of the commercial gerotor pumps adopt the curtate epitrochoid curves (i.e. $\mu>1$ ) to avoid self-intersection (or undercutting) phenomenon.

In Eqs. (4), $\delta_{t}$ represents the tip clearance. This tip clearance is usually inevitable due to many practical considerations such as, precision of machining tools, prevention of jamming conditions, noise reduction, and application of lubricants, etc.

The result for the profile of the outer-rotor is omitted here for the limitation of the space. For further information, consult the Ref. (3).

\section{Non-undercut condition on the inner-rotor}

As is well known, the formula for the radius of curvature of a parametric curve is

$$
\rho=\frac{\left[\left(x^{\prime}\right)^{2}+\left(y^{\prime}\right)^{2}\right]^{3 / 2}}{x^{\prime} y^{\prime \prime}-x^{\prime \prime} y^{\prime}}
$$

where $x$ and $y$ are coordinates of the parametric curve, $x^{\prime}, x^{\prime \prime}$ and $y^{\prime}, y^{\prime \prime}$ are the first and the second derivatives of $x$ and $y$ with respect to the parameter, respectively.

If the mesh points of ${ }^{3 f} C$ in Eqs. (4) are differentiated with respect to $\phi$, then the resulting formula for the radius of curvature of the tooth profile of the inner-rotor will be very complicated because of the term of $\psi$. It will be impossible to obtain directly an explicit formula. However, it will be overcome by the introduction of the radius of curvature of the point $N_{i}$, i.e. when $\delta_{t}=-R_{r}$. After some calculation, it yields a simple formula for the radius of curvature $\rho$ of the inner-rotor as follows:

$$
\frac{\rho}{R}=\frac{\left[\mu^{2}+1-2 \mu \cos (N-1) \phi\right]^{\beta / 2}}{\mu\left[\mu^{2}+N-\mu(N+1) \cos (N-1) \phi\right]^{-\lambda}}-\lambda
$$

where $\lambda=R_{r} / R$ is the tooth size ratio.

To demonstrate the interference (or undercut) phenomenon, two inner-rotors are depicted simultaneously in Fig. 2. The same design parameters $(R=6, N=5, E=1)$ have been used in Fig. 2, but with the exception of $R_{r}$ (equal to 2 in the larger rotor and 3 in the smaller rotor). 


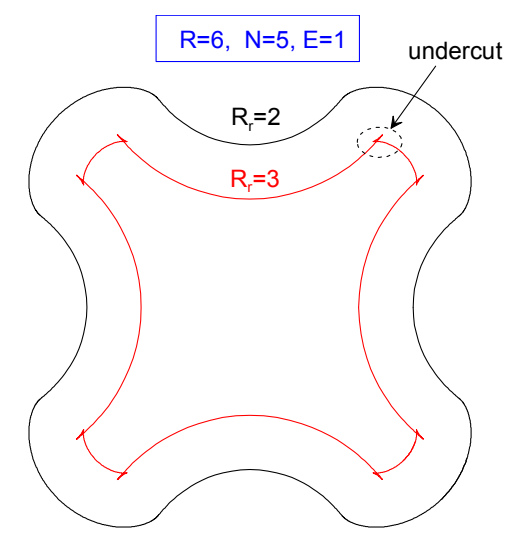

Fig.2 Inner-rotor profile design example for showing undercut

Shown in Fig. 3 is the relationship between radius of curvature $\rho$ of the tooth profile and the generated parameter $\phi_{2}$. From Fig. 3, we can observe that the radius $R_{r}$ of the cylindrical outer-rotor teeth increases, then $\rho$ decreases. If $R_{r}$ is larger than a limit value, the $\rho_{\min }$ on convex section will be negative and the tooth profile of the inner-rotor will be self-intersecting. This will produce backlash between the inner-rotor and outer-rotor teeth during running.

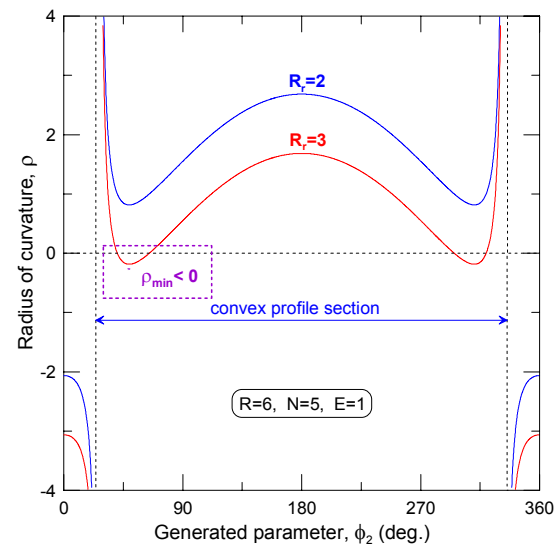

Fig.3 Radius of curvature for the design example

To avoid this undercut, the point with zero radius of curvature must be avoided, i.e. the minimum value of $\rho$ of the tooth profile on convex section should not be less than zero. Setting $d \rho / d \phi=0$, we can obtain an explicit formula for calculating the maximum value of $\lambda$ of the outer-rotor tooth size ratio to avoid undercut on the inner-rotor in the form:

$$
\lambda_{\max }=\frac{\left(R_{r}\right)_{\max }}{R}=\frac{1}{\mu}\left(\frac{3}{N+1}\right)^{3 / 2} \sqrt{\left(\mu^{2}-1\right)(N-1)}
$$

Using Eq. (7), it is very easy to calculate limit dimension. For an example, if the design parameters are given as $R=6, N=5$, and $E=1$, the maximum outer-rotor tooth radius to avoid undercutting is $\left(R_{r}\right)_{\max }=2.34521$.

\section{Hertzian contact stress}

The chamber volume reaches either maximum or minimum values when it is symmetrical over the $x_{3 f}$-axis. Two contact points form the seals that separate the 
high-pressure fluid of the outlet port and low-pressure fluid of the inlet port. Thus, once all coordinates of the contact points are known, we can calculate the fluid moment $T_{i n}$ on the internal rotor about $\mathrm{O}_{3}$. The force caused by the fluid pressure will have the components $-p \Delta y$ and $-p \Delta x$, and it acts through the midpoint of the line joining the two contact points.

Since there are several contact points, the problem of calculating the contact forces (concurrent force system at the pitch point $I_{23}$ ) is statically indeterminate (Fig. 4).
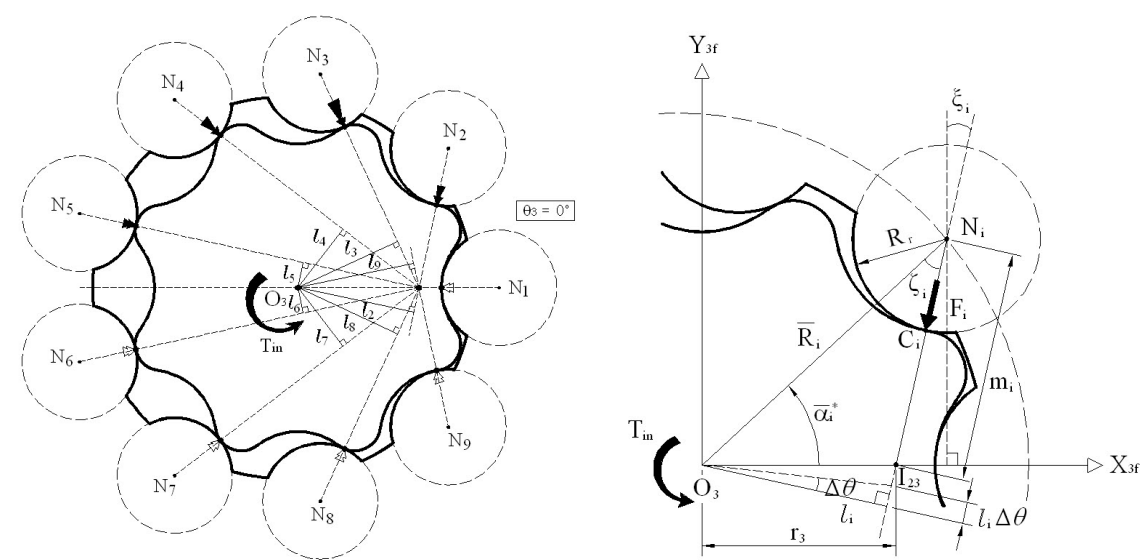

Fig.4 Distribution of contact forces

The method used here to find the contact forces is as follows: It is assumed that the bearings of both rotors are stiff compared with the stiffness $k_{e}$ of the teeth. Thus, when a couple is applied to the inner-rotor, it will rotate a small amount $\Delta \theta$ about its axis, until the tooth deformations $\delta_{i}\left(=l_{i} \Delta \theta\right)$ are sufficiently large to provide forces whose combined moments about $O_{3}$ are equal to $T_{i n}$. At any instant about half of the teeth will have contact forces which contribute to the moment $T_{i n}$ while at the remaining teeth a small separation opens up, and there is in fact no contact. Black arrows shown in Fig. 4 represent the magnitude range of the contact force for the reference position, while white arrows represent no force.

If the input torque $T_{i n}$ about $O_{3}$ is given, the contact force to the inner-rotor $F_{i}$ at any tooth $N_{i}$ can be found from the condition of moment equilibrium with the aid of the Palmgren relation $^{(4)}\left(F_{i}=k_{e} \delta_{i}^{n}\right.$ where $\left.n=10 / 9\right)$ as follows:

$$
F_{i}=\frac{T_{i n} l_{i}^{n}}{\sum_{i=1}^{N} l_{i}^{n+1}}
$$

where the value of $l_{i}$ can be calculated in the form

$$
l_{i}=\eta_{i} \frac{r_{3} \bar{R}_{i}}{m_{i}} \sin \bar{\alpha}_{i}^{*}
$$

and $\eta_{i}$ is the active zone discrimination parameter, it has 1 for $0<\bar{\alpha}_{i}^{*}<\pi$ but 0 for $\pi \leq \bar{\alpha}_{i}^{*} \leq 2 \pi$. The geometric definitions of $\bar{R}_{i}, m_{i}$ and $\bar{\alpha}_{i}^{*}$ are found in Ref. (3).

Knowing the normal force at contact point, it is possible to evaluate the maximum 
Hertzian contact stress $p_{H}$ as follows:

$$
p_{H}=\sqrt{\frac{F_{i} E^{*}}{2 \pi H R^{*}}}
$$

where $H$ is the rotor thickness, $E^{*}$ is the reduced modulus of elasticity, and $R^{*}=\left(1 / \rho_{i}+1 / R_{r}\right)^{-1}$ is the composite radius of curvature, respectively. In this relation the value of the radius of curvature $\rho_{i}$ is determined by using the Euler-Savary equation ${ }^{(5)}$ in the form:

$$
\rho_{i}=\left(m_{i}-I_{23} N_{i}^{\prime}\right)-R_{r}
$$

where $I_{23} N_{i}^{\prime}$ can be found as

$$
\frac{1}{r_{3}}-\frac{1}{r_{2}}=\left(\frac{1}{m_{i}}-\frac{1}{I_{23} N_{i}^{\prime}}\right) \sin \xi_{i}
$$

\section{Quasi-static FE analysis}

The pump shown in Fig. 5 (in-house developed MFC program) has proportions similar to pumps that are commercially available. The pump with the rotor thickness of $H=9.25$ $\mathrm{mm}$ was analyzed, using the values of $R=40.725 \mathrm{~mm}, R_{r}=10.85 \mathrm{~mm}, N=9$ and eccentricity $E=2.85 \mathrm{~mm}$. Here the radius of the circle that contains the outer-rotor profile was set as $R_{c}=35.8 \mathrm{~mm}$ (see Fig. 1). The specific volume (the theoretical displacement) is evaluated as $9.74 \mathrm{~cm}^{3} / \mathrm{rev}$. and determined using the following formula

$$
V_{t h}=\Delta A(N-1) H
$$

where $\Delta A$ is equal to the difference of the maximum chamber area and the minimum chamber area.
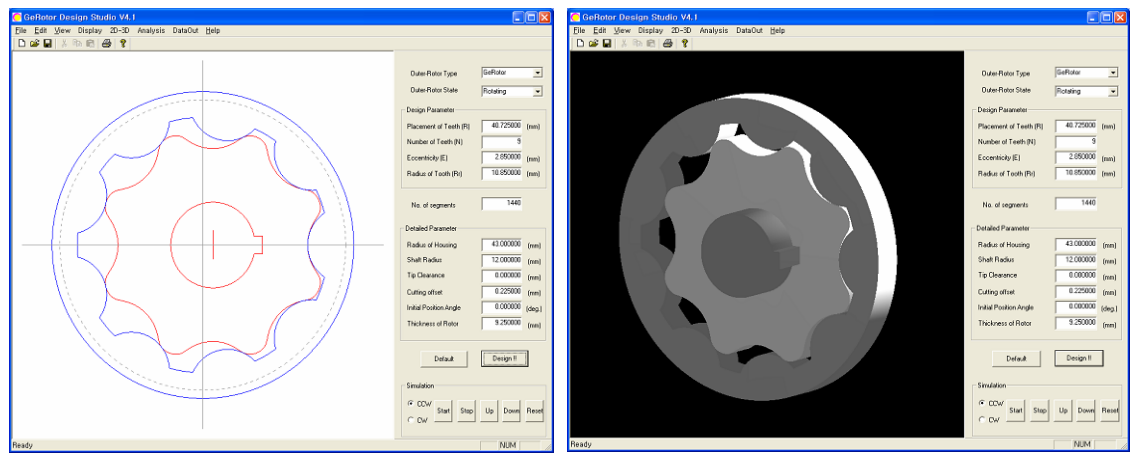

Fig.5 Simulation screen for the kinematic analysis

Simulation work is now taken under study for the above specific unit. It does not take into account the manufacturing, clearance and thermal effects in this preliminary approach. The profiles of the rotors have to be defined very accurately in order to obtain a correct geometry of the assembled rotor set. Initial penetration has to be avoided because a virtual interference would appear producing unreal results. For this approach, we have used the commercial FEA package of ANSYS. 
Figure 6 shows the FE mesh of the assembled rotor set. The elements have been more refined on the contact points. A small rotation of $\Delta \theta$ is given to the inner-rotor using MPC184 element and the contact stress at the contact point is calculated by the penalty of the penetration caused in each tooth. The analysis has been carried out in a quasi-static mode without taking into account of the dynamic components in the deformation equations.

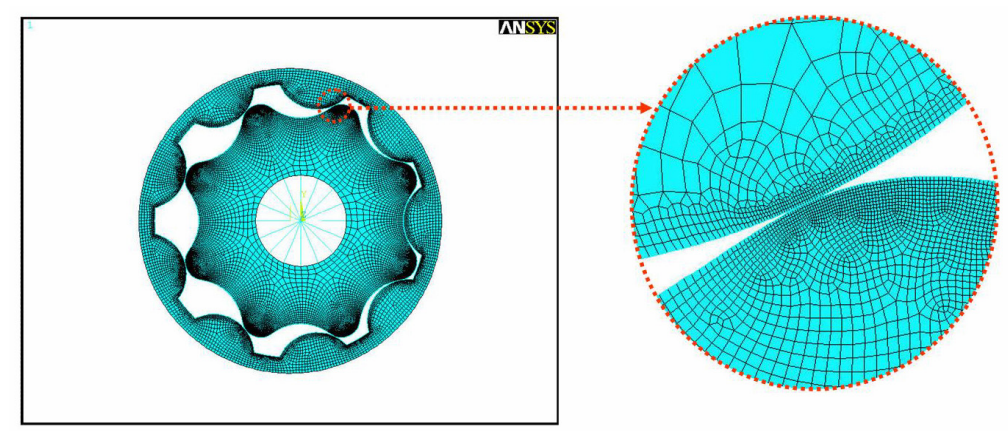

Fig.6 FE mesh result

Figure 7 shows an example of the analysis, and the maximum result of von-Mises stress is $96.5 \mathrm{MPa}$ at the mesh point of the $3^{\text {rd }}$ outer-rotor tooth while $92.2 \mathrm{MPa}$ via Eq. (11). The input torque of $2 \mathrm{Nm}$ was applied on the center of the inner-rotor at the rotation position of $\theta_{3}=0$. Also the selected material properties were $E=210 \mathrm{GPa}$ and $v=0.29$ for all the rotors. The results shown are three contact points while the analytical study shown are four. One may need a more sophisticated the geometric modeling, being more accurate and to increase the mesh density. Simulation work continues and it will be able to obtain more results for modeling the crankshaft and housing of the pump.

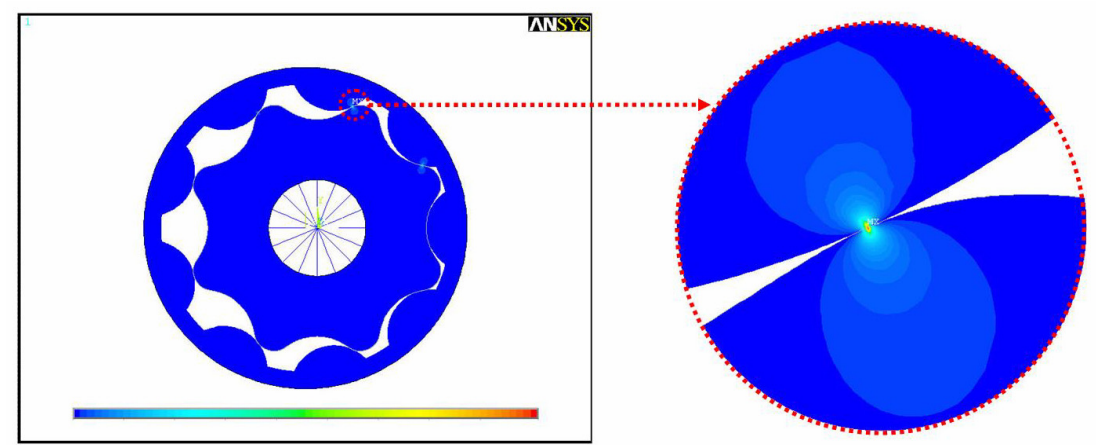

Fig.7 Post-processing result (nodal solution: von-Mises stress)

\section{Wear rate proportional factor}

Based on experimental data of various unlubricated (dry) material pairs, the vast majority being metallic, it is possible to write the laws of adhesive wear as follows. The amount of wear is generally proportional to the applied load and the sliding distance and generally inversely proportional to the hardness of the surface being worn away. For a designer who is interested in the rate of wear depth, the wear rate is proportional to the $p_{H} V_{s}$ factor (here $V_{s}$ is the sliding velocity) or the life of an interface is inversely proportional to the $p_{H} V_{s}$ factor. The factor of $p_{H} V_{s}$ in this paper is referred to as the wear rate proportional factor (WRPF).

The sliding velocity at the meshing point of $C_{i}$ between the $i$-th outer-rotor tooth and the inner-rotor can be found of the form 


$$
V_{s}=I_{23} C_{i}\left(\omega_{3}-\omega_{2}\right)=\left(\frac{m_{i}-R_{r}}{N}\right) \omega_{3}
$$

where $\omega_{3}$ and $\omega_{2}$ are the angular velocities of the inner and outer-rotors, respectively.

The normalized sliding velocity $V_{s} / \omega_{3}$ and WRPF $p_{H} V_{s} / \omega_{3}$ of the tooth at the $i$-th outer-rotor $N_{i}$ with the variation of the position of the outer-rotor are shown in Fig. 8. Here we presented only 5 results out of total 9 for graphical brevity.
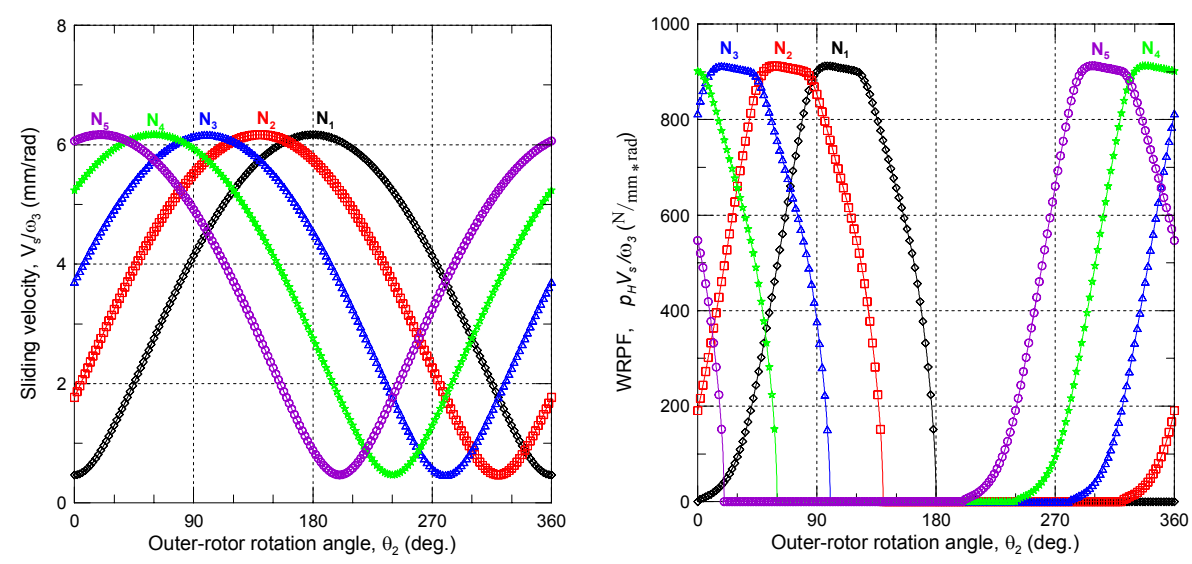

Fig.8 Sliding velocity and WRPF with the rotation of the outer-rotor

It reveals that the sliding velocity arrives at the maximum value when the outer rotor lies on negative $x_{3 f}$-axis (e.g., $\theta_{2}=\pi$ for $N_{1}$ tooth) or the minimum chamber volume appears. However, the maximum wear position is expected at shift rotation position before arriving at the maximum sliding position. This WRPF on the milestone work will be generally used as a relative wear performance index for the selection of the rotor set material pairs and the geometric parameters.

\section{Conclusions}

Results of the contact stress of the analytical method, FE modeling and simulation are presented for a specific unit of a gerotor pump. The knowledge of the contact stress obtained by both methods gives an optimum exactness to take into consideration the results and to continue this work. Finally, analytical method has been presented to calculate the WRPF to a rotor set when it works as a part of an internal gear pump type gerotor.

\section{References}

(1) Colbourne, J.R., 1976. Reduction of the contact stress in internal gear pumps. Trans. ASME, Journal of Engineering for Industry, pp. 1296-1300.

(2) Shin, J.-H. and Kwon, S.-M., 2006. On the lobe profile design in a cycloid reducer using instant velocity center. Mechanism and Machine Theory, Vol. 41, No. 5, pp. 596-616.

(3) Kim, J.C., 2007. "A study on tooth-profile design in a trochoid-gear pump." Master's Thesis (in Korean), Changwon National University, Republic of Korea.

(4) Palmgren, A., 1967. Les Roulements. Description, Theorie, Applications (SKF Compagnie d'Applications Mecaniques).

(5) Shigley, J.E. and Uicker, Jr. J.J. Theory and Machines and Mechanisms. McGraw-Hill, 1980. 